Citation information:

B. D. Geraedts, C. M. Arndt, and A. M. Steinberg, "Rayleigh Index Fields in Helically Perturbed Swirl-Stabilized Flames Using Doubly Phase Conditioned $\mathrm{OH}^{\star}$

Chemiluminescence Tomography", Flow, Turbulence and Combustion 96(4):1023-38

(2016)

The final publication is available at Springer via:

http://dx.doi.org/10.1007/s10494-016-9727-4 


\title{
Rayleigh Index fields in helically perturbed swirl-stabilized flames using doubly phase conditioned $\mathrm{OH}^{*}$ chemiluminescence tomography
}

\author{
B. D. Geraedts - C. M. Arndt • A. M. \\ Steinberg
}

Received: date / Accepted: date

\begin{abstract}
This paper demonstrates a method for calculating thermoacoustic energy transfer (viz. Rayleigh Index) fields in complex swirl-stabilized flames having asymmetric 3D flow structures using high-repetition-rate $\mathrm{OH}^{*}$ chemiluminescence measurements. Measurements were acquired in a variety of perfectly premixed methane-air flames, each of which contained a helical velocity disturbance that was coupled with a precessing vortex core (PVC). The azimuthal position of the PVC and helical disturbance relative to the viewing angle was determined by tracking the position of the chemiluminescence centoid. Tomographic reconstruction of multiply-phase-conditioned mean chemiluminescence fields then was performed to determine the mean 3D shape of the helically-perturbed heat release field at different phases over the thermoacoustic cycle. These fields, in combination with measured pressured signals, allowed
\end{abstract}

B. D. Geraedts

Institute for Aerospace Studies

University of Toronto

4925 Dufferin Street

Toronto ON

Canada M3H 5T6

C. M. Arndt

Institute of Combustion Technology

German Aerospace Center (DLR)

38-40 Pfaffenwaldring

70569 Stuttgart

Germany

A. M. Steinberg

Institute for Aerospace Studies

University of Toronto

4925 Dufferin Street

Toronto ON

Canada M3H 5T6

E-mail: adam.steinberg@utoronto.ca 
calculation of the thermoacoustic energy transfer distribution. Complex patterns were found, which generally involved considerable energy transfer in the periphery of the burner (i.e. towards the outer recirculation zone). The total energy transfer was found to scale with the limit-cycle oscillation amplitude. This method provides a relatively simple and robust diagnostic for determining combustor regions driving thermoacoustic oscillations.

Keywords Thermoacoustic instability · Combustion dynamics · Swirl flames · Chemiluminescence · Tomography

\section{Introduction}

Thermoacoustic oscillations in gas turbine combustors remain a major issue of practical concern [1]. As described by Rayleigh [2], the oscillation amplitudes of coupled heat release rate $(\dot{q})$ and pressure $(p)$ oscillations in a combustor grow if the rate of energy transfer from unsteady combustion to the pressure field exceeds the rate at which acoustic energy is dissipated or transmitted $(D)$. The magnitude of thermoacoustic oscillations are referred to as the Rayleigh field $(\Psi)$ and the relationship between these is provided in Eq. 1, which describes the integration of the product of fluctuating components of pressure $\left(p^{\prime}\right)$ and heat release rate $\dot{q}^{\prime}$ integrated in time $t$ and volume $v$

$$
\Psi=\int_{v} \psi(\mathbf{x}) \mathrm{d} \mathbf{x}=\frac{\gamma-1}{\gamma} \frac{1}{\bar{p}} \int_{v} \int_{t} \dot{q}^{\prime}(\mathbf{x}, t) p^{\prime}(\mathbf{x}, t) \mathrm{d} t \mathrm{~d} \mathbf{x}
$$

where $\gamma$ is the ratio of specific heats. Limit-cycle amplitudes are reached when non-linear behavior results in $\Psi=D$, at which point the oscillations may be sufficient to cause considerable fatigue stress, reduced efficiency, or combustor extinction due to blow-off or flashback. High values of $\Psi$ are associated with regions of high local energy transfer, $\psi$, which depends on the local amplitude and relative phase of the $\dot{q}^{\prime}$ and $p^{\prime}$ oscillations; $\psi$ positive if the local oscillations are less than $90^{\circ}$ out-of-phase.

At present, it is not possible to predict either the $\psi$ distribution or total $\Psi$ in practical gas turbine combustors due to the complexity of the underlying flow-flame-acoustic interactions. Gas turbine combustors generally employ high degrees of swirl to generate recirculation zones (due to vortex breakdown) near the combustor exit, and thereby provide a low-speed region for robust flame anchoring. Such swirling jets often manifest hydrodynamic instabilities, the most common of which is a single helical mode accompanied by a precessing vortex core (HPVC) $[1,3]$. This results in a helical velocity disturbance that rotates around the combustor at a constant Strouhal number, and alter the $\dot{q}$ distribution. For notational convenience, the combination of helical mode and PVC will be referred to as the HPVC. The helical nature of the flame in such situations has been clearly demonstrated by Stohr et al. using $\mathrm{OH}$ planar laser induced fluorescence (PLIF) [4], Moeck et al. using $\mathrm{OH}^{*}$ chemiluminescence tomograpahy [5], and others [1]. 
In a perfectly premixed swirl-stabilized combustor, Steinberg et al. [6] and Oberleithner et al. [7] showed a direct correspondence between the presence of a HPVC and high-amplitude limit-cycle oscillations. Moreover, Arndt et al. [8] showed that transition from a flow with symmetric vortex shedding to one with a HPVC preceded transition from low- to high- amplitude oscillations. This clearly indicates that the helical nature of the flow and flame leads to thermoacoustically-coupled interactions that are conducive to regions of high $\psi$. To elucidate such interactions, it is necessary to determine the helically disturbed heat release rate field and how it oscillates over the thermoacoustic cycle. This is complicated by the inherently 3D and dynamic (i.e. precessing) nature of the disturbance. Large eddy simulations (LES) have been able to predict helical modes and oscillation frequencies in swirl stabilized flames (e.g Refs. $[9,10,11,12])$, however there are many open questions regarding the ability of LES to predict limit-cycle thermoacoustic oscillations and coupling mechanisms due to model uncertainty, boundary conditions, etc.

To gain insight regarding the 3D flow and flame structure and the associated dynamics, Steinberg et al. [13, 14] and Caux-Brisebois et al. [15] resolved multi-kHz repetition-rate OH PLIF and stereoscopic-PIV measurements with respect to the angular position of the HPVC relative to the measurement plane. A proper orthogonal decomposition (POD) was applied to the planar 3-component velocity fields obtained to determine a reference signal corresponding to the HPVC oscillation signal. Offline phase-conditioning was subsequently applied to obtain a mean velocity field and mean flame surface density field (derived from OH-PLIF) at a set of discrete phase angles $\left(\phi_{h}=1 \ldots N_{h}\right)$ over the mean cycle of the reference signal. Thermally coupled dynamics could then be understood by applying a second phase-conditioning using the acoustic pressure fluctuations in the combustion chamber as the reference signal, $\phi_{a}=1 \ldots N_{a}$. A planar field representing the coupling between FSD and pressure could then be obtained for each $\phi_{h}$ by integrating over the acoustic cycle. Interpolating between the planar fields then results a fully 3D approximation of the $\psi$ fields. It was found that $\psi$ exhibited a distinctly helical structure due to relative axial motion of the HPVC and flame over the thermoacoustic cycle. Thermoacoustic coupling in the burner periphery (i.e. the outer recirculation zones) was found to be critical in setting the limit-cycle amplitude [15].

While the aforementioned studies have clarified many interesting aspects of thermoacoustic oscillations, the planar laser diagnostics employed present many challenges for application in practical high-pressure combustors due to restricted optical access, laser reflections, and broadening of spectral lines. In such systems, imaging of the natural chemiluminescence emissions from excited $\mathrm{OH}^{*}$ or $\mathrm{CH}^{*}$ radicals is a more readily applied diagnostic. These excited states are result in light emission throughout the $3 \mathrm{D}$ reaction zone and give a reasonably accurate picture of the heat release rate, particularly for perfectly premixed methane-air flames $[16,17]$. Thus, camera-based measurements of the light emissions at the spectral lines associated with these species result in a line-of-sight integrated projection of the $3 \mathrm{D}$ flame. For a single image, an 


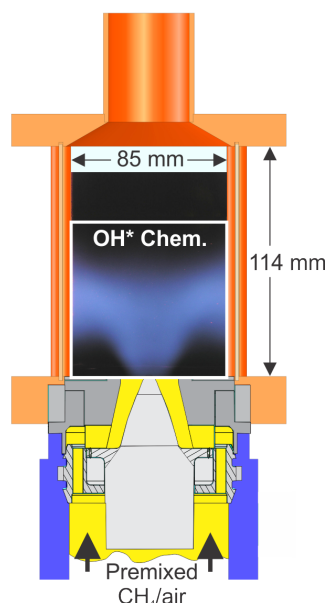

Fig. 1 Combustor schematic with typical flame image.

Abel deconvolution yields a pseudo-spatially resolved mean 2D cross-section, assuming that the flame is axisymmetric [18].

For flames with non-axisymmetric features of interest, a tomographic reconstruction can be used to obtain spatially resolved information from CL measurements. Tomography techniques aim to determine the internal geometry of a radiation emitting or absorbing body based on multiple projections (views) of path integrated information through that body at different angles. Tomographic reconstruction of chemiluminescence images has been performed by several authors in both laminar and turbulent flames, e.g Refs. [19, 20]. Instantaneous reconstructions can be performed by simultaneously taking images from several viewing angles (typically greater than 10 for good results), whereas reconstructions of mean fields or stable laminar flames often are obtained by rotating a single camera around the target and taking images at numerous angles[21].

In a system with a rotating helical disturbance, Moeck et al. [5] demonstrated that tomographic reconstruction of the helically deformed mean heat release rate field could be performed using a single viewing angle and phaseconditioned average measurements, with the phase reference signal representing the angular position of the HPVC relative to the viewing angle. In such a system, the phase-conditioned mean chemiluminescence fields represent different views of the mean helically deformed heat release rate field; instead of rotating the imager around a static source, the source rotates on an axis with respect to the imager. Several reconstruction algorithms and phase-resolution methods were tested, which resulted in different reconstruction accuracies in artificial phantom studies, but similar general results. This is only applicable to combustors with axisymmetric geometries, and for more complex combustor geometries, a multi-view and/or multi camera

The present work uses double phase-conditioning similar to Caux-Brisebois et al. [15] to perform tomographic reconstruction of the mean helically de- 


\begin{tabular}{cccccccc} 
Case & $\varphi$ & $P_{\mathrm{th}}[k W]$ & $\dot{m}_{\mathrm{air}}[\mathrm{g} / \mathrm{min}]$ & $\dot{m}_{\mathrm{CH} 4}[\mathrm{~g} / \mathrm{min}]$ & $p^{\prime \prime}[\mathrm{Pa}]$ & $f_{\mathrm{a}}[H z]$ & $f_{\mathrm{h}}[H z]$ \\
\hline \hline 4 & 0.7 & 25 & 733 & 30.0 & 74.4 & 306 & 578 \\
$5 \mathrm{a}$ & 0.65 & 30 & 940 & 36.0 & 149.1 & 289 & 706 \\
$5 \mathrm{~b}$ & 0.7 & 30 & 880 & 36.0 & 145.4 & 314 & 693 \\
6 & 0.7 & 35 & $1.02 \times 10^{3}$ & 42.0 & 181.9 & 380 & 799
\end{tabular}

Table 1 Experimental cases, mass flow rates $(\dot{m})$ of air and fuel, root-mean-squared pressure fluctuation amplitude $\left(p^{\prime \prime}\right)$, dominant thermo-acoustic frequency $\left(f_{\mathrm{a}}\right)$, and rotation frequency of the $\operatorname{HPVC}\left(f_{\mathrm{h}}\right)$.

formed heat release rate field at each phase in the thermoacoustic cycle for four perfectly premixed swirl-stabilized flames, each of which exhibited self-excited limit-cycle thermoacoustic oscillations of different amplitude. This allows calculation of $3 \mathrm{D} \psi$ fields based entirely on chemiluminescence measurements.

\section{Experiment and Diagnostics}

The basic combustor geometry employed for this work, shown in Fig. 1, has been the subject of numerous previous experimental and computational studies $[6,10,15,22,23]$. Fuel $\left(\mathrm{CH}_{4}\right)$ and air were combined in a commercial premixer located approximately $2 \mathrm{~m}$ upstream of the plenum. The mixture then passed through a plate with a choked orifice, located in a $25 \mathrm{~mm}$ diameter tube $0.15 \mathrm{~m}$ upstream of the combustor plenum entrance. This configuration isolated the fuel and air supply from the combustor acoustics and provided known upstream boundary conditions.

The $\mathrm{CH}_{4}$ /air mixture was fed from the $25 \mathrm{~mm}$ diameter tube into the combustor plenum ( $78 \mathrm{~mm}$ diameter, $100 \mathrm{~mm}$ length), through a radial swirler (12 swirl vanes), to the burner nozzle (27.85 $\mathrm{mm}$ exit diameter), and then into the combustion-chamber $(85 \times 85 \mathrm{~mm}$ cross-section, $114 \mathrm{~mm}$ length $)$. The inner surface of the nozzle was formed by a conical bluff body. The combustionchamber itself was composed of four large fused-silica windows, held in the corners by Inconel posts. The large windows provided adequate optical access to the combustion chamber, allowing for the application of optical diagnostics. Further information on the combustor geometry can be found in Ref. [23].

The overall experimental program consisted of nine conditions, covering a range of thermal powers $(10-35 \mathrm{~kW})$ and equivalence ratios $(\varphi=0.65-$ 0.80 ). Here, we focus on four cases exhibiting different amplitude oscillations, which are described in Table 1. The naming scheme for the cases is chosen to be consistent with previous analysis of the flames using PLIF and PIV measurements performed by Steinberg et al. and Brisebois et al. [6, 15]. All flow rates were controlled using electromechanical mass flow controllers (Brooks) and monitored using Coriolis mass flow meters (Siemens Sitrans F C) with an uncertainty of $1.5 \%$. The integral length scale is set by the nozzle exit radius, and the resultant characteristics place these flames in the corrugated flamelet regime of turbulent premixed combustion.

Combustion chamber and plenum had multiple ports for pressure and temperature measurements. Pressures were measured using calibrated micro- 
phone probes (Brüel \& Kjaer, Type 4939), sampled at a rate of $50 \mathrm{kHz}$. The combustion-chamber pressure was measured using two probes in a corner post, and the plenum pressure was measured using a probe flush with the plenum wall.

The pressure measurements were recorded simultaneously with $\mathrm{OH}^{*}$ Chemiluminescence measurements. The line-of-sight integrated spontaneous emission of electronically excited $\mathrm{OH}\left(\mathrm{OH}^{*}\right)$ in the spectral range around $310 \mathrm{~nm}$ was imaged at $10 \mathrm{kHz}$ repetition-rate using a high-speed camera (LaVision HSS5) equipped with an external, two-stage, lens-coupled intensifier (LaVision HSIRO) and a $45 \mathrm{~mm} \mathrm{f} / \#=1.8 \mathrm{UV}$ lens (Cerco). The intensifier gate time was set to $15-25 \mu \mathrm{s}$ (depending on conditions) in order to capture sufficient signal. Background luminosity and elastic scattering were reduced by using a hightransmission ( $>80 \%$ at $310 \mathrm{~nm}$ ) bandpass interference filter. The field-of-view was $85 \times 85 \mathrm{~mm}$, spanning the entire combustor width and covering well beyond the axial location of the flame. At $10 \mathrm{kHz}$, the camera sensor had $512 \times 512$, $20 \mu \mathrm{m}$ square active pixels. Light emitted from the flame was connected along a conical path having a divergence angle of about $7^{\circ}$. Intensifier non-uniformity was corrected based on the measured white-field response. A minimum of two independent data acquisitions were conducted at each condition, resulting in over 15000 individual measurements of a given flame.

Simultaneous $10 \mathrm{kHz}$ S-PIV, OH PLIF, and $\mathrm{OH}^{*}$ chemiluminescence measurements were obtained using the high-repetition-rate diagnostics system at the German Aerospace Center Institute for Combustion Technology. The SPIV and OH PLIF systems are not used explicitly in this work and details are given in Refs. [6, 15].

At each condition, the power spectrum of the pressure signal showed one dominant frequency $\left(f_{a}\right)$ that is listed in Table 1 . The total chemiluminescence in the combustion chamber also oscillated at this frequency, indicating that this is the mode of self-excited thermoacoustic oscillations. Detailed characterization using multiple pressure measurement ports and Comsol simulations have shown that the system operates as a Helmholtz resonator [24]. Hence, the pressure oscillations were essentially spatially uniform throughout each of the combustion and plenum chambers, with significant spatial pressure gradients only within the swirler/nozzle assembly and exhaust tube. The HPVC frequency $\left(f_{h}\right)$ in Table 1 was determined from the motion of the $\mathrm{OH}^{*}$ chemiluminescence field, as described below.

\section{Analysis techniques}

Three major steps are required to convert the temporal sequence of $\mathrm{OH}^{*}$ chemiluminescence measurements to $3 \mathrm{D}$ fields showing the helical flame disturbance and its oscillations over the thermoacoustic cycle:

1. Phase-conditioned averaging of the measurements with respect to both the angle of the helical disturbance (relative to the camera viewing angle) and the thermoacoustic cycle. 


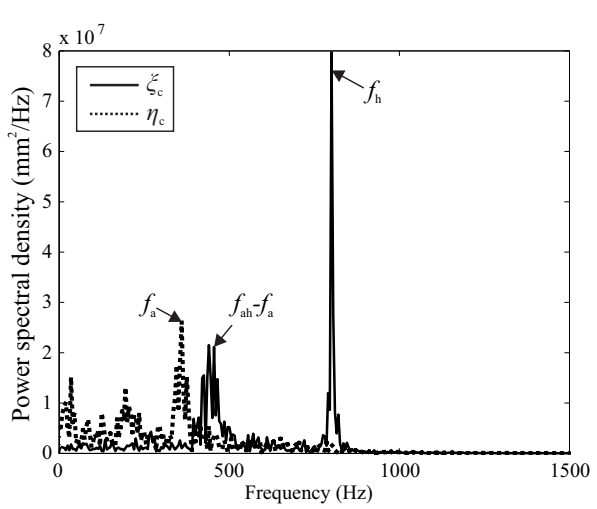

Fig. 2 Power spectral density of chemiluminescence image centroid motion for Case 6.

2. Tomographic reconstruction of the mean 3D chemiluminescence field at each phase in the thermoacoustic cycle.

3. Calculation of the Rayleigh Integral (Eq. 1)

\subsection{Phase conditioned averaging}

Offline phase-conditioning is a commonly used analysis technique, in which measurements are obtained simultaneously with a reference signal. Each oscillation of the reference signal is divided into a discrete number of phases, $\phi=1 \ldots N$, and each instantaneous measurement assigned to the phase number at which the measurement occurred. Conditional averages may then be taken at each phase. For the present analysis, two reference signals are required to identify the phase in the combustion chamber pressure oscillation and the angular position of the HPVC. In the latter case, this signal was taken directly from the calibrated microphone probes. As discussed in Section 2, spatial variations in the combustion chamber pressure were negligible, and thus assumed to be spatially invariant. The pressure field cam thus be described by

$$
p(\mathbf{x}, t) \approx p(t)=\bar{p}+p^{a}\left(\phi_{a}\right)+p^{t}(t)
$$

where $p^{a}$ represents the coherent oscillation in the pressure field at $f_{a}, p^{t}$ is a stochastic effect representing cycle-to-cycle variability, $\phi_{a}=1 \ldots N_{a}$ is the phase through the thermoacoustic cycle, and $N_{a}$ is the number of phases used for the conditioning. For the purpose of thermoacoustic energy transfer analysis (viz. Eq. 1), $p^{a}$ is the relevant quantity. Statistical considerations described below required that the thermoacoustic cycle be discretized into only $N_{a}=6$ phases.

Determining a reference signal for the phase, i.e. angular position, in the HPVC cycle requires post processing of the measurements. In previous work, the reference signal was obtained from temporal coefficients arising through POD of multi-kHz repetition-rate S-PIV data $[13,14,15]$. These temporal 


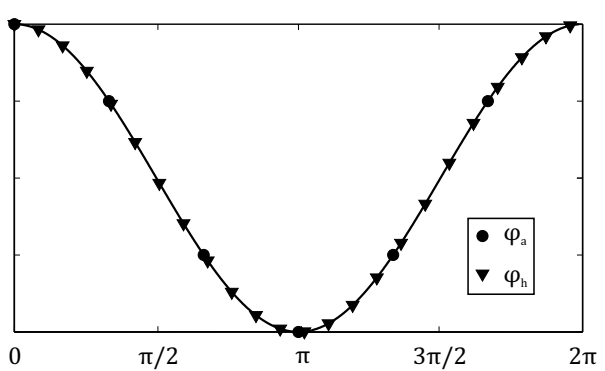

Fig. 3 Location of phase angles used for conditioning with respect to acoustic and helical disturbance cycles.

coefficients typically were single-mode signals that were periodic over one rotation of the HPVC around the combustor. The objective here is to obtain an equivalent reference signal based entirely on the $\mathrm{OH}^{*}$ chemiluminescence data.

The overall chemiluminescence intensity in the combustor oscillated at $f_{a}$, showing that the heat release rate was coupled to the acoustic oscillations. It previously has been shown that the motion of the HPVC around the combustion chamber causes a rotational motion of the flame that results in detectable signals at $f_{h}[13,15,25]$. In the present data, it was observed that the projected chemiluminescence images underwent a distinct transverse motion that had a helical signature.

An instantaneous chemiluminescence image can be described as $\xi(\zeta, \eta, t)$, where $\xi$ and $\eta$ are the image coordinates in the horizontal and vertical directions, respectively. To describe the bulk flame dynamics in these images, the motion of the intensity-weighted projected chemiluminescence centroid $\left(\zeta_{c}(t), \eta_{c}(t)\right)$ was calculated. Power spectra of these signals are shown in Fig. 2 for Case 6 . The vertical motion oscillated at $f_{a}$ due to axial motion of the flame over the thermoacoustic cycle. However, the horizontal motion oscillated at a different frequency, which was identical to that of the HPVC calculated by POD of the velocity field in Ref. [15]. This correspondence was found for all cases, indicating that $\xi_{c}(t)$ can be used as the $f_{h}$ reference signal for phase resolution with respect to the helical disturbance. It is noted that a secondary peak occurred in the $\xi_{c}$ spectrum at $f_{h}-f_{a}$.

Each instantaneous chemiluminescence image can then be described as

$$
\xi(\zeta, \eta, t)=\bar{\xi}(\zeta, \eta)+\xi^{a h}\left(\zeta, \eta, \phi_{a}, \phi_{h}\right)+\xi^{t}(\zeta, \eta, t)
$$

where $\xi^{a h}$ represents the double-phase-conditioned mean, $\phi_{h}=1 \ldots N_{h}$ is the angle of the HPVC as determined from the $\zeta_{c}$ reference signal (relative to an arbitrary angle), and $\xi^{t}$ is the stochastic fluctuation. At a fixed $\phi_{a}$, changes in $\xi^{a h}$ with $\phi_{h}$ represent different mean projections of the chemiluminescence field as seen when the HPVC is at different angles relative to the detector.

For each case, 15,740 images were acquired. The pressure reference signal was divided into $N_{a}=6$ phases to resolve across the thermoacoustic cycle, 


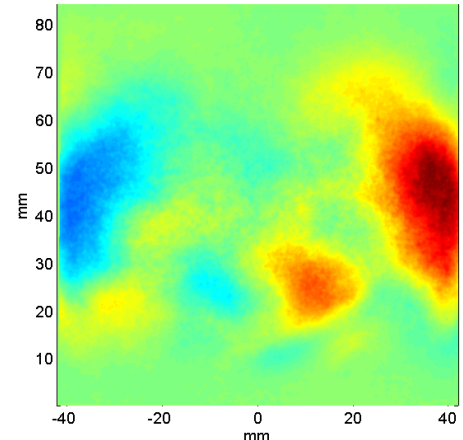

(a) $\phi_{a}=1, \phi_{h}=1$

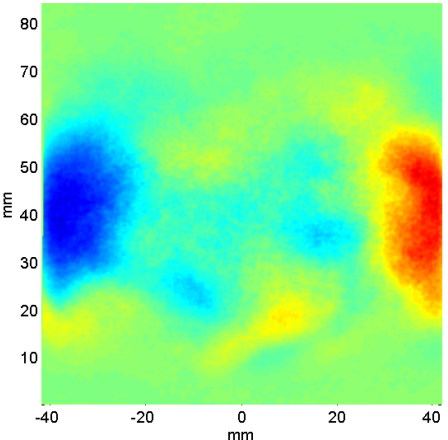

(c) $\phi_{a}=4, \phi_{h}=1$

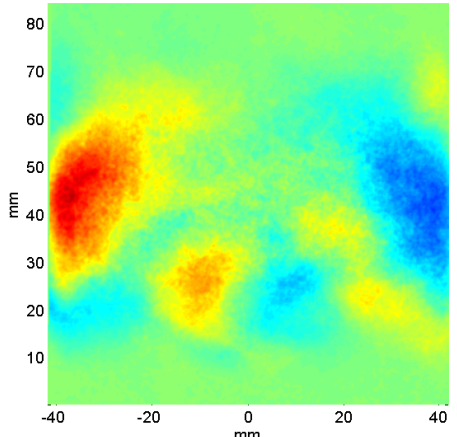

(b) $\phi_{a}=1, \phi_{h}=12$

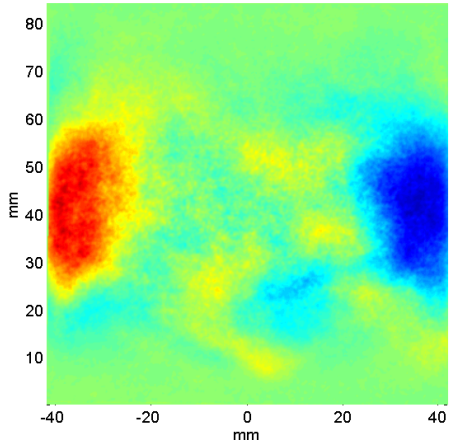

(d) $\phi_{a}=4, \phi_{h}=12$

Fig. 4 Variations of doubly-phase-conditioned mean chemiluminescence images $\left(\xi^{a h}\right)$ across the thermoacoustic cycle $\left(\phi_{a}\right)$ and rotation of the HPVC $\left(\phi_{h}\right)$ for Case 6 .

resulting in approximately 2600 images at each $\phi_{a}$. These images were then conditioned based on $\zeta_{c}$ into $N_{h}=23$ phases, representing 23 different views of the helical heat release perturbation field at each $\phi_{a}$. The resultant ca. 115 images at each phase angle combination were sufficient to provide good statistical convergence. The use of 23 phases prevents any two phase-conditioned mean fields that are exactly mirrored from each other, which was found to produce better reconstructions. The phases used for this work are shown in Fig. 3 and the procedure for selecting the number of phases is discussed below.

Figure 4 shows $\xi^{a h}$ fields across variations in $\phi_{h}$ at constant $\phi_{a}$, and across $\phi_{a}$ at constant $\phi_{h}$ for Case 6 . As can be seen, $\phi_{h}$ is associated with changes in the projected chemiluminescence in the $\zeta$ direction as the disturbance rotates, whereas $\phi_{a}$ is primarily associated with changes in the chemiluminescence intensity over the thermoacoustic cycle. Note that the fields at $\phi_{h}=1$ and 12 are not separated by exactly $180^{\circ}$; the images therefore are not exactly mirrored across the vertical axis. 


\subsection{Tomographic reconstruction of chemiluminescence}

Tomographic reconstruction techniques for chemiluminescence are well established in the literature, and only a few pertinent details are discussed here. A Tomographic reconstruction of a 3D object begins with a series of $2 \mathrm{D}$ projections, $\Gamma_{\alpha}(\boldsymbol{\xi})$, where $\boldsymbol{\xi}$ represents the coordinates of the sensor in 2 dimensions and $\alpha$ indicates the angle at which the object is being viewed. The result of performing the tomographic reconstruction is the field $\Sigma(\mathbf{x})$, where $\mathbf{x}$ represents the $3 \mathrm{D}$ coordinates of the combustor volume. As in this work, combustion studies typically utilize a camera mounted perpendicularly to the combustor axis that collect the line-of-sight integrated emissions from the flame. Hence, $\Gamma=\xi^{a h}, \boldsymbol{\zeta}=(\zeta, \eta)$, and $\alpha=\phi_{h}$. The reconstructed $\Sigma(\mathbf{x})$ then correspond to to a reconstructed $3 \mathrm{D}$ heat release field, $\tilde{q}^{a}(\mathbf{x})$. A common assumption in chemiluminescence tomography is that all rays striking the sensor are parallel. However, this is not strictly true for typical camera imaging, wherein entocentricity results in cone-beam effects.

Common techniques to achieve tomographic reconstructions include the method of filtered back projection and closely related methods in Fourier space [26]. While relatively computationally inexpensive, these transform-based techniques assume a perfect scanner and highly simplified physics, leading to numerical artifacts and high susceptibility to noise. Furthermore, these techniques require a large number of projections [5].

Algebraic reconstruction techniques (ART) are more computationally expensive algorithms, but overcome some of the issues with filtered back projection [27]. In ART, the reconstruction problem is treated by solving a system of linear algebraic equations. For a parallel beam configuration, a square grid is imposed over the reconstruction domain. The unknowns in the system correspond to the values in each cell of the grid, the algebraic relationships to the contribution of each pixel to the path integral along a ray (based on the projection geometry), and the solution array to projected (line integral) pixel values. High resolution tomography uses a large number of grid points, making direct inversion very computationally expensive and typically requiring iterative methods. Despite the computational expense, ART algorithms have shown to have favorable performance in configurations with a limited number of views or low signal-to-noise. ART algorithms also can be constructed for non-parallel beam configurations.

A slight modification to ART algorithms is the simultaneous iterative reconstruction technique (SIRT). In a typical ART algorithm, the solution for each unknown is updated individually as it is computed, and therefore affects the solution for all subsequent unknowns. Conversely, SIRT algorithms update the entire solution array simultaneously. This typically results in less noise and better looking reconstructions.

Implementations for all of these algorithms are readily available for a variety of programming languages. Here, the ASTRA Toolbox for Matlab from the University of Antwerp has been used to perform the reconstructions [28]. This toolbox includes GPU-accelerated tomography routines that allow rapid pro- 
cessing of high resolution fields. Moreover, cone-beam algorithms are included that account for the non-parallel light rays inherent to camera imaging.

While the SIRT technique applied here requires a higher computational load and is more susceptible to noise than a tailored reconstruction technique, it does not require any prior assumptions about the spatial structure of the fields to be reconstructed $[5,26]$. Thus, SIRT techniques are potentially more widely applicable to flame structures with more complex features than a single circumferential Fourier component. With a sufficient number of views, SIRT techniques can eliminate noise, and furthermore calculation using GPUaccelerated algorithms running on consumer-grade graphics cards can reduce the computation time to manageable levels.

Different tomography algorithms were tested to determine the reconstruction quality for relevant configurations. To do so, an imaging phantom was created with a single helical mode of the form

$$
f(r, \theta, z)=\left\{\begin{array}{lr}
P(r, \varphi, z)=\sin (2 \pi r) \cdot \cos (\varphi+z \cdot 2 \pi) \cdot(\sin (z \cdot \pi))^{2}: & r \leq R \\
0 & r \geq R
\end{array}\right.
$$

and shown in Fig. 5. This phantom, similar in form to the one used by Moeck et al. [5] was chosen to be used in place of the standard Shepp-Logan phantom because of its similarity to the expected chemiluminescence fields and its continuous variations in intensity, which can be more difficult to reconstruct with a low number of projection angles. The projections of this phantom were calculated based on a conical beam viewing configuration corresponding to the imaging optics employed in the experiment. Reconstructions were performed using ART, SIRT and SIRT with 3D cone-beam correction algorithms, the latter of which attempts to correct for the imperfect entocentricity inherent in using camera lens-based (non-parallel rays) imaging.

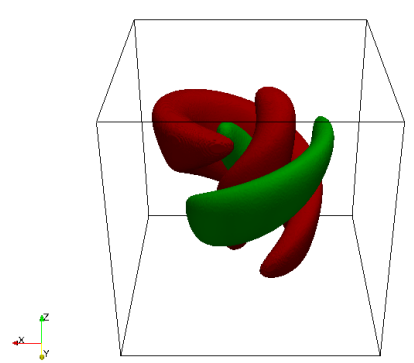

(a) Original Phantom

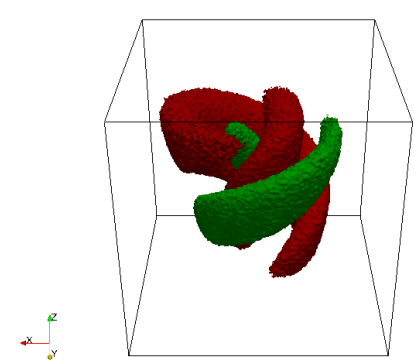

(b) SIRT Reconstruction

Fig. 5 Isosurfaces of original flame phantom (a) and reconstruction of phantom using a cone-beam SIRT reconstruction algorithms. Isosurfaces are shown at $30 \%$ (green) and $-30 \%$ (red) of maximum of continuous distribution flame phantom. 


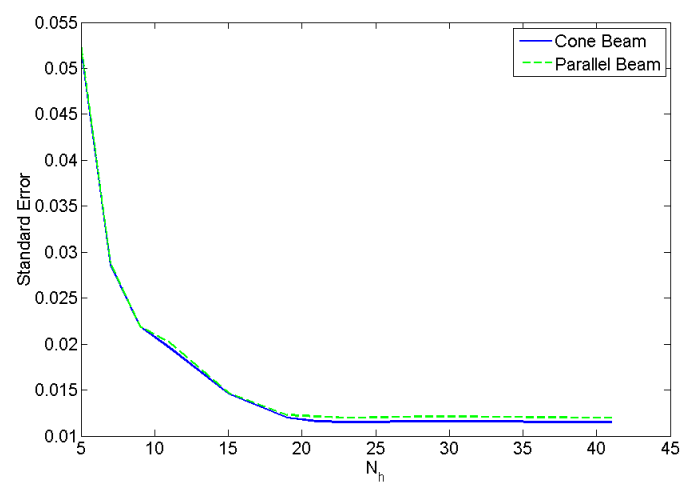

Fig. 6 Standard error of the reconstructions of the flame phantom using cone-beam and parallel-beam SIRT reconstruction techniques.

The accuracy of the CT reconstructions produced using different values of $N_{h}$ (from 5 to 40) was quantified by the spatially averaged error $\langle\epsilon\rangle$ of the reconstructed $\tilde{P}(\mathbf{x})$ when compared to the original $P(\mathbf{x})$, as

$$
\langle\epsilon\rangle=\sum_{\mathbf{x}} \frac{|P(\mathbf{z})-\tilde{P}(\mathbf{x})|}{|P(\mathbf{x})|}
$$

These results are shown using cone beam and parallel beam reconstruction methods in Fig. 6. It was found that the error asymptoted to the minimum of 0.0115 , or $4 \%$ of the mean value of the phantom, at $N_{h}=23$ when using the cone-beam technique. This is slightly lower error than the error using a parallel beam reconstruction techniques. Therefore, it was concluded that for this data set $N_{h}=23$ phase angles were to be used when reconstructing the experimental data for all cases, and a cone-beam reconstruction algorithm that attempts to account for non-parallel line-of-sight measurements would provide the best result. For each tomographic reconstruction using 23 phase angles, approximately 11 minutes were required to compute the $3 \mathrm{D}$ reconstruction from the set of projection images

Using $N_{h}=23$ HPVC phase angles, the number of acoustic phase angles, $N_{a}$, that could be used was determined by calculating the standard error of the mean $\sigma_{\bar{\xi}}$, averaged over $(\zeta, \eta)$ when successive $\xi(\zeta, \eta, t)$ measurements, $n_{s}$, were added to each $\xi^{a h}\left(\zeta, \eta, \phi_{h}, \phi_{a}\right)$ phase-conditioned mean field. In this way, the minimum required instantaneous measurements could be determined by finding the number that are required to reduce $\operatorname{Var}\left(\xi^{a h}\right)$ to less than $5 \%$ of the spatially averaged mean of $\xi^{a h}\left(\zeta, \eta, \phi_{h}, \phi_{a}\right)$. Approximately 50 samples were required to reduce the variance to less than $5 \%$ of the mean value, as shown in Fig. 7. It was concluded from these results that the double phase averaged result $\xi^{a h}\left(\zeta, \eta, \phi_{h}, \phi_{a}\right)$ at each $\left(\phi_{h}, \phi_{a}\right)$ combination must have at least 50 images included, and this was obtained using $N_{a}=6$ acoustic phase angles. 


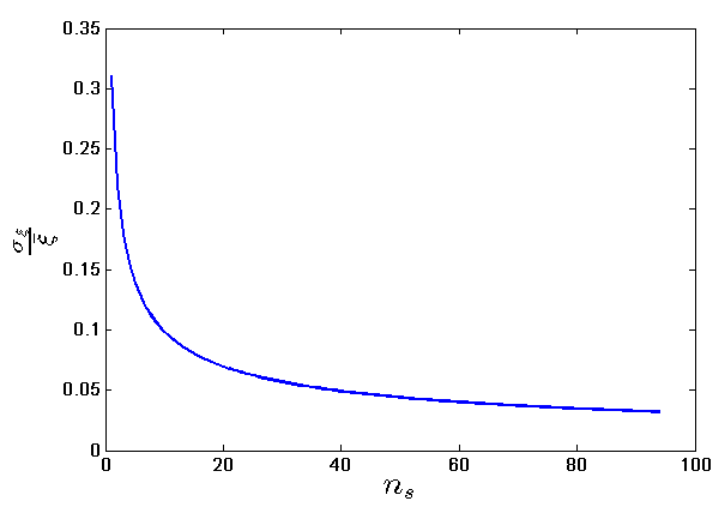

Fig. 7 Variance of $\xi\left(\phi_{a}=1, \phi_{h}=1\right)$ as a fraction of spatially averaged mean fields with successive samples for case $5 \mathrm{~b}$.

\subsection{Thermoacoustic energy transfer}

The tomographic reconstructions described above result in 3D heat release oscillation fields at each $\phi_{a}$ in the thermoacoustic cycle, viz. $q^{a}\left(x, y, z, \phi_{a}\right)$. Cartesian coordinates are used instead of the cylindrical system in Eq. 4, since these are the coordinates produced by the SIRT algorithm. From these fields, the local thermoacoustic energy transfer was calculated as

$$
\psi(x, y, z) \sim \int_{\phi_{a}} \tilde{q}^{a}\left(x, y, z, \phi_{a}\right) p^{a}\left(\phi_{a}\right) \mathrm{d} \phi_{a}
$$

and the total energy transfer, $\Psi$, as the integral of $\psi$ over the measurement volume.

\section{Results}

Figure 8 shows the reconstructed $q^{a}$ fields for Case $5 \mathrm{~b}$ at each phase in the thermoacoustic cycle. These results are downsampled by a factor of 3 to reduce the high-frequency noised believed to be primarily due to the noise in the original doubly-phase-conditioned mean chemiluminescence fields. This downsampling potentially smears out some small-scale features in the reconstructions, but this could be improved in future works by achieving better convergence in the $\xi^{a h}\left(\zeta, \eta, \phi_{h}, \phi_{a}\right)$ fields using larger initial data sets. The reconstructions are restricted to the region more than $5 \mathrm{~mm}$ from the chamber walls, since reflections adversely affected the accuracy of the CL signals close to the walls.

In these results, two primary regions of interest can be observed. Towards the center of the combustor volume exists an inner $q^{a}$ region exhibiting a distinct helical shape due to the influence of the HPVC. In this region, both positive and negative perturbations exist. Over the thermoacoustic cycle, the 


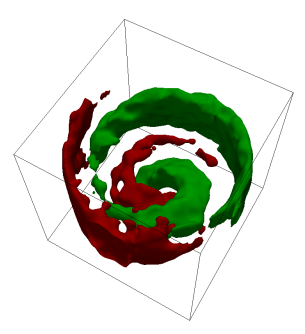

(a) $\phi_{a}=1$

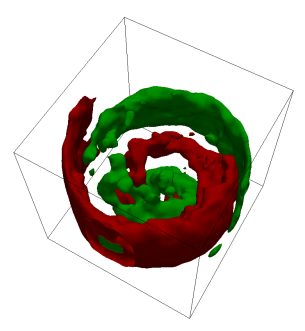

(d) $\phi_{a}=4$

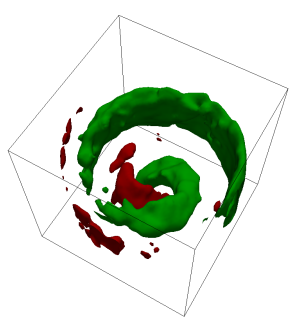

(b) $\phi_{a}=2$

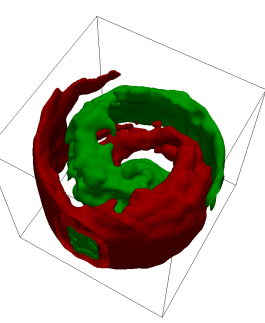

(e) $\phi_{a}=5$

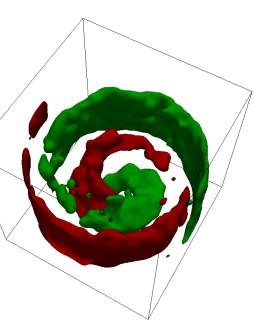

(c) $\phi_{a}=3$

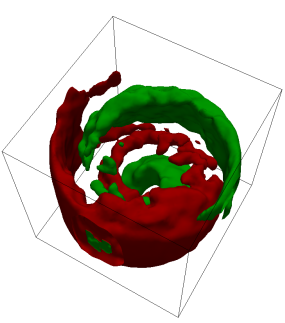

(f) $\phi_{a}=6$

Fig. 8 Reconstructions of CL fields over acoustic cycle for case 5b. Green and red isosurfaces are positive and negative $q^{a}$, respectively, at $50 \%$ of the maximum $q^{a}$ amplitude across all phases.

region of negative $q^{a}$ decreases in size (increasing heat release) as the pressure decreases, whereas the positive $q^{a}$ region has oscillations in size that are relatively in-phase with the pressure. This indicates that oscillations in heat release in this inner region are roughly in-phase with the pressure oscillations, indicating positive coupling in this region.

Towards the outer region of the combustor exists a toroidal region of heat release oscillations, with opposed positive and negative regions on the upper right and lower left sides of each subfigure in Fig. 8. The size of the positive and negative regions fluctuated to a much larger degree than in the inner helical region over the $\phi_{a}$ cycle, suggesting that this region contributed more significantly to the total thermoacoustic coupling than the inner region. Furthermore, it appears that at $\psi_{a}=5$ that the inner and outer regions are connected, suggesting a continuous expanding helical structure at this instance. The opposed inner and outer positive and negative regions observed in these reconstructions are consistent with the observations of Caux-Brisebois et al. [15] obtained using PLIF and PIV diagnostics on the same flames.

The $\psi$ fields that result from integrating the product of the reconstructed CL fields and pressure oscillation over the acoustic cycle are shown in Fig. 9 for all cases. Once again, these fields feature high levels of noise that obscure more detailed features due to the limitations of the CT reconstructions. However, the dominant behavior and trends are clear. For example, Fig. 10 shows the total $\Psi$ versus the root-mean-squared pressure oscillations for all four cases. The observed positive correlation is in approximate agreement observations 


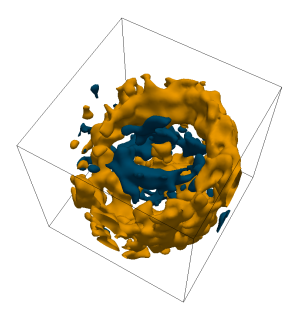

(a) Case 4

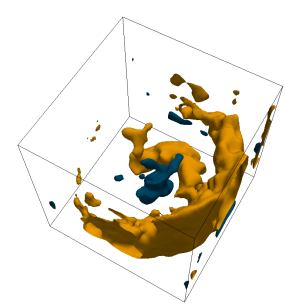

(c) Case $5 \mathrm{~b}$

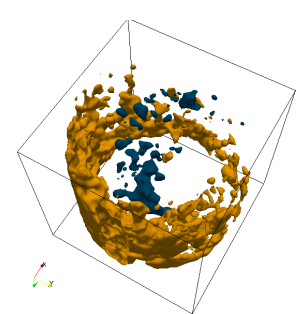

(b) Case 5 a

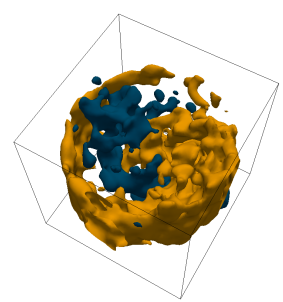

(d) Case 6

Fig. $9 \psi$ fields for cases $4,5 \mathrm{a}, 5 \mathrm{~b}$ and 6 . Yellow and blue isosurfaces are positive and negative $\psi$, respectively, at $50 \%$ of the maximum positive $\psi$ amplitude

by Caux-Brisebois et al [15]. However, the higher $\Psi$ observed for case 5 a at approximately the same acoustic oscillation amplitude of case $5 \mathrm{~b}$ suggests a non-linear relationship, and the change in measured $\Psi$ may be dependent on the different equivalence ratios between these two cases.

The $\psi$ fields for each case exhibit inner and outer thermoacoustic coupling regions. In all cases, the outer region is dominated by positive $\psi$. In the inner region, all cases feature both positive and negative coupling, with cases 4 and 5 a showing stronger negative than positive $\psi$ regions in the inside. These observations are consistent with the $q^{a}$ oscillations described for Case 5b in Fig. 8. Cases with higher limit-cycle amplitudes exhibit larger regions of positive $\psi$ in the combustor periphery. Hence, the phase delay between periodic combustion in this region and the combustion chamber pressure is critical for determining the thermoacoustic behavior of the combustor.

\section{Conclusion}

This paper has demonstrated a relatively simple technique for determining thermoacoustic energy transfer fields in complex swirl-stabilized flames having 3D coherent vortex structures (i.e. HPVCs). By phase conditioning the measurement based on the HPVC azimuthal position relative to the measurement plane and the phase in the thermoacoustic cycle, mean projections of the helically perturbed heat release field could be obtained and several viewing angles at different combustor pressures. From these, the phase-conditioned mean 3D helically perturbed heat release rate field at different phases over the 


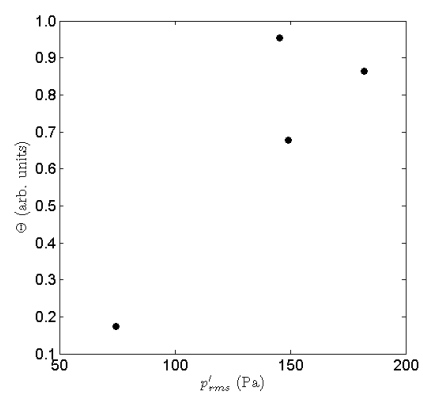

Fig. 10 Total $\Psi$ for cases $4,5 \mathrm{a}, 5 \mathrm{~b}$, and 6 showing linear correlation between Thermoacoustic energy transfer and pressure oscillation.

thermoacoustic oscillation was determined by tomographic reconstruction, and the consequent thermoacoustic energy transfer.

The tomographically reconstructed fields clearly show the helical heat release perturbation caused by the HPVC and how this changed over the thermoacoustic cycle. Moreover, thermoacoustically coupled heat release oscillations in the outer recirculation zones, similar to those observed using laser diagnostics [15]. The total calculated energy transfer increased with the measured limit-cycle pressure oscillation amplitude, providing further support for the accuracy of the method. This technique provides a new means of determining how thermoacoustic oscillations are excited in practical combustors, which may not be amenable to laser diagnostics.

Acknowledgements This work was supported by NSERC Canada under grants RGPIN 413232 and STPGP 43036

\section{References}

1. Huang, Y., Yang, V.: Dynamics and stability of lean-premixed swirlstabilized combustion. Prog. Energy Combust. Sci. 35, 293-365 (2009)

2. Rayleigh, J.S.W.: The Theory of Sound. Dover, New York (1945)

3. Syred, N.: A review of oscillation mechanisms and the role of the precessing vortex core (PVC) in swirl combustion systems. Prog. Energy Combust. Sci. 32, 93-161 (2006)

4. Stöhr, M., Sadanandan, R., Meier, W.: Phase-resolved characterization of vortex-flame interaction in a turbulent swirl flame. Exp. Fluids 51(4), 1153-1167 (2011)

5. Moeck, J.P., Bourgouin, J.F., Durox, D., Schuller, T., Candel, S.: Tomographic reconstruction of heat release rate perturbations induced by helical modes in turbulent swirl flames. Experiments in fluids 54(4), 1-17 (2013)

6. Steinberg, A.M., Arndt, C.M., Meier, W.: Parametric study of vortex structures and their dynamics in swirl stabilized combustion. Proc. Combust. Inst. 34, 3117-3125 (2013) 
7. Oberleithner, K., Stöhr, M., Im, S.H., Arndt, C.M., Steinberg, A.M.: Formation and flame-induced suppression of the precessing vortex core in a swirl combustor: experiments and linear stability analysis. Combust. Flame 162(8) (2014)

8. Arndt, C.M., Steinberg, A.M., Boxx, I.G., Meier, W., Aigner, M., Carter, C.D.: Flow-field and flame dynamics of a gas turbine model combustor during transition between thermo-acoustically stable and unstable states. Proceedings of the ASME Turbo Expo GT2010-22830, 677-687 (2010)

9. Duwig, C., Fuchs, L.: Large eddy simulation of vortex breakdown/flame interaction. Phys. Fluids 19, 075,103 (2007)

10. Franzelli, B., Riber, E., Gicquel, L.Y.M., Poinsot, T.: Large eddy simulation of combustion instabilities in a lean partially premixed swirled flame. Combust. Flame 159, 621-637 (2012). DOI 10.1016/j.combustflame.2011.08.004

11. Lieuwen, T.: Modeling premixed combustion-acoustic wave interactions: A review. Journal of Propulsion and Power 19(5), 765-781 (2003)

12. Roux, S., Lartigue, G., Poinsot, T., Meier, U., Bérat, C.: Studies of mean and unsteady flow in a swirled combustor using experiments, acoustic analysis and large eddy simulations. Combust. Flame 141, 40-54 (2005)

13. Steinberg, A.M., Boxx, I., Stöhr, M., Carter, C.D., Meier, W.: Flowflame interactions causing acoustically coupled heat release fluctuations in a thermo-acoustically unstable gas turbine model combustor. Combust. Flame 157, 2250-2266 (2010)

14. Steinberg, A.M., Boxx, I., Stöhr, M., Meier, W., Carter, C.D.: Effects of flow structure dynamics on thermoacoustic instabilities in swirl-stabilized combustion. AIAA J. 50(4), 952-967 (2012)

15. Caux-Brisebois, V., Steinberg, A.M., Arndt, C.M., Meier, W.: Thermoacoustic velocity coupling in a swirl stabilized gas turbine model combustor. Combust. Flame 161, 3166-3180 (2014)

16. Najm, H., Paul, P., Mueller, C., , Wyckoff, P.: On the adequacy of certain experimental observables as measurements of flame burning rate. Combust. Flame 113, 312-332 (1998)

17. Lauer, M., Sattelmayer, T.: On the adequacy of chemiluminescence as a measure for heat release in turbulent flames with mixture gradients. J. Eng. Gas Turb. Power 132, 061,502 (2010)

18. Palies, P., Durox, D., Schuller, T., Candel, S.: The combined dynamics of swirler and turbulent premixed swirling flames. Combustion and Flame 157(9), 1698-1717 (2010)

19. Floyd, J., Kempf, A.M.: Computed tomography of chemiluminescence (ctc): High resolution and instantaneous 3-d measurements of a matrix burner. Proc. Comb. Inst. 33, 751-758 (2011)

20. Li, X., Ma, L.: Capabilities and limitations of 3d flame measurements based on computed tomography of chemiluminescence. Combust. Flame 10.1016/j.combustflame.2014.08.020 (2014)

21. Worth, N., Dawson, J.: Tomographic reconstruction of oh* chemiluminescence in two interacting turbulent flames. Meas. Sci. Technol. 24, 024,013 
(2013)

22. Weigand, P., Meier, W., Duan, X.R., Aigner, M.: Laser-based investigations of thermoacoustic instabilities in a lean premixed gas turbine model combustor. J. Eng. Gas Turb. Power 129, 664-671 (2007)

23. Meier, W., Weigand, P., Giezendanner-Thoben, R.: Detailed characterization of the dynamics of thermoacoustic pulsations in a lean premixed swirl flame. Combust. Flame 150(1), 2-26 (2007)

24. Seong, H.I.: Caratrisation des instabilits thermoacoustiques dans les chambres de combustion gaz. Ph.D. thesis, École Centrale de Paris (2012)

25. Shtork, S., Vieira, N., Fernandes, E.: On the identification of helical instabilities in a reacting swirling flow. Journal of Propulsion and Power 19(5), 765-781 (2003)

26. Deans, S.R.: The Radon transform and some of its applications. Courier Dover Publications (2007)

27. Kak, A.C., Slaney, M.: Principles of computerized tomographic imaging. Society for Industrial and Applied Mathematics (2001)

28. Palenstijn, W.J., Batenburg, K.J., Sijbers, J.: Performance improvements for iterative electron tomography reconstruction using graphics processing units (gpus). J. Struct. Biol. 176, 250-253 (2011) 